\title{
Particle-number projection method in time-dependent Hartree-Fock theory: Properties of reaction products
}

\author{
Kazuyuki Sekizawa, ${ }^{1}$ and Kazuhiro Yabana ${ }^{1,2,0}$ \\ ${ }^{1}$ Graduate School of Pure and Applied Sciences, University of Tsukuba, Tsukuba 305-8571, Japan \\ ${ }^{2}$ Center for Computational Sciences, University of Tsukuba, Tsukuba 305-8577, Japan
}

(Dated: April 26, 2018)

\begin{abstract}
Background: The time-dependent Hartree-Fock (TDHF) theory has been successful in describing low-energy heavy ion collisions. Recently, we have shown that multinucleon transfer processes can be reasonably described in the TDHF theory combined with the particle-number projection technique.
\end{abstract}

Purpose: In this work, we propose a theoretical framework to analyze properties of reaction products in TDHF calculations.

Methods: TDHF calculation in three-dimensional Cartesian grid representation combined with particle number projection method.

Results: We develop a theoretical framework to calculate expectation values of operators in the TDHF wave function after collision with the particle-number projection. To show how our method works in practice, the method is applied to ${ }^{24} \mathrm{O}+{ }^{16} \mathrm{O}$ collisions for two quantities, angular momentum and excitation energy. The analyses revealed following features of the reaction: The nucleon removal proceeds gently, leaving small values of angular momentum and excitation energy in nucleon removed nuclei. Contrarily, nuclei receiving nucleons show expectation values of angular momentum and excitation energy which increase as the incident energy increases.

Conclusions: We have developed a formalism to analyze properties of fragment nuclei in the TDHF theory combined with the particle-number projection technique. The method will be useful for microscopic investigations of reaction mechanisms in low-energy heavy ion collisions as well as for evaluating effects of particle evaporation on multinucleon transfer cross sections.

\section{INTRODUCTION}

The time-dependent Hartree-Fock (TDHF) theory has been successfully applied for studying low-energy nuclear reactions: fusion reactions, deep inelastic collisions, quasi-fission reactions, extraction of nucleus-nucleus potentials and dissipation coefficients, and so on (for a recent review, see Ref. [1]). For reactions producing projectile- and target-like fragments, expectation values of operators are often evaluated in the TDHF wave function after collision to investigate properties of produced nuclei.

Recently, a particle number projection (PNP) technique has been proposed by C. Simenel [2]. This method has made it possible to calculate transfer probabilities efficiently from the TDHF wave function after collision and has been successfully applied [3 5]. Using the PNP technique, we studied multinucleon transfer (MNT) processes in heavy ion reactions at around the Coulomb barrier for several systems [5] for which extensive measurements are available [6 9]. Comparing calculated cross sections with the measurements, we have concluded that the TDHF theory may describe MNT cross sections quantitatively, in an accuracy comparable to calculations by other existing theories such as GRAZING [10] and Complex WKB [11], which are based on semiclassical approximation, and a model based on Langevin-type equations of motion [12, 13].

\footnotetext{
* sekizawa@nucl.ph.tsukuba.ac.jp
}

† yabana@nucl.ph.tsukuba.ac.jp
The PNP technique has been utilized to calculate reaction probabilities which are required to calculate cross sections of specific nucleon numbers. To investigate reaction mechanisms, it will be useful to calculate expectation values of operators in the particle-number projected wave functions. This is the subject of this article. We will consider a general method to calculate expectation values of one- and two-body operators.

The method will be also useful to investigate deexcitation effects on the MNT cross sections. The produced nuclei through MNT reactions are often highly excited. Measured cross sections are affected by deexcitation processes such as particle emissions which take place in relatively longer timescale. In the existing theories mentioned above, evaporation effects are usually taken into account employing statistical models. In statistical models of particle evaporation, excitation energy and angular momentum are the basic inputs. The method to be developed in this paper will be useful to calculate these quantities.

In nuclear structure calculations, it is a routine work to calculate expectation values of operators in particlenumber projected wave functions [14]. In this article, we extend the formalism to the TDHF wave function after collision with the PNP. To illustrate how our method works, we analyze properties of produced nuclei in ${ }^{24} \mathrm{O}+{ }^{16} \mathrm{O}$ collisions.

This article is organized as follows. In Sec. III we describe a general formalism to calculate expectation values of operators in the TDHF wave function after collision with the PNP. In Sec. III, we apply the method to ${ }^{24} \mathrm{O}+{ }^{16} \mathrm{O}$ collisions, as an illustrative example. In 
Sec. IV a summary is presented.

\section{FORMULATION}

\section{A. Particle-number projection method}

We consider microscopic TDHF calculations of lowenergy heavy ion collisions in which two fragments, a projectile-like fragment (PLF) and a target-like fragment (TLF), are produced. In this section, we develop a general formalism to calculate expectation values of operators for one of the fragments, either PLF or TLF, with the PNP. We first describe the formalism assuming that the system is composed of $N$ identical fermions. An extension to include two kinds of fermions, neutrons and protons, is straightforward.

We assume that the fragments are well separated spatially after collision at the final stage of the TDHF calculation. We define two spatial regions, $V$ and $\bar{V}$. The spatial region $V$ includes a fragment to be analyzed. $\bar{V}$ is the complement of $V$, which includes the other fragment.

We denote the TDHF wave function after collision as $\Psi\left(x_{1}, \cdots, x_{N}\right)$, where $x$ denotes a set of the spatial and the spin coordinates, $x \equiv(\mathbf{r}, \sigma)$. The wave function $\Psi$ is, in general, not an eigenstate of the particle-number operator in the spatial region $V$ but a superposition of states with different particle numbers in $V$. It can be expressed as

$$
\Psi\left(x_{1}, \cdots, x_{N}\right)=\sum_{n=0}^{N} \Psi_{n}\left(x_{1}, \cdots, x_{N}\right),
$$

where $\Psi_{n}$ denotes a particle-number projected wave function,

$$
\Psi_{n}\left(x_{1}, \cdots, x_{N}\right)=\hat{P}_{n} \Psi\left(x_{1}, \cdots, x_{N}\right) .
$$

$\Psi_{n}$ is a component of $\Psi$ having $n$ particles in the spatial region $V$ and $N-n$ particles in the spatial region $\bar{V}$. The operator $\hat{P}_{n}$ is the PNP operator defined by [2, $[$ ]

$$
\begin{aligned}
\hat{P}_{n} & =\sum_{s\left(\left\{\tau_{i}\right\}: V^{n} \bar{V}^{N-n}\right)} \Theta_{\tau_{1}}\left(\mathbf{r}_{1}\right) \cdots \Theta_{\tau_{N}}\left(\mathbf{r}_{N}\right) \\
& =\frac{1}{2 \pi} \int_{0}^{2 \pi} d \theta e^{\mathrm{i}\left(n-\hat{N}_{V}\right) \theta},
\end{aligned}
$$

where $s\left(\left\{\tau_{i}\right\}: V^{n} \bar{V}^{N-n}\right)$ indicates that a sum over the sequence $\tau_{1} \tau_{2} \cdots \tau_{N}$ should be taken for all possible combinations that $V$ appears $n$ times and $\bar{V}$ appears $N-n$ times. We have introduced a space division function, $\Theta_{\tau}(\mathbf{r})$, and a particle-number operator in the spatial region $\tau, \hat{N}_{\tau}$, which are defined by

$$
\Theta_{\tau}(\boldsymbol{r})=\left\{\begin{array}{lll}
1 & \text { if } \boldsymbol{r} \in \tau, \\
0 & \text { if } \boldsymbol{r} \notin \tau,
\end{array}\right.
$$

and

$$
\hat{N}_{\tau}=\int_{\tau} d \mathbf{r} \sum_{i=1}^{N} \delta\left(\mathbf{r}-\mathbf{r}_{i}\right)=\sum_{i=1}^{N} \Theta_{\tau}\left(\mathbf{r}_{i}\right),
$$

where $\tau$ represents the spatial region either $V$ or $\bar{V}$.

We consider a general operator $\hat{\mathcal{O}}$ and decompose it into two operators according to the spatial regions:

$$
\hat{\mathcal{O}}=\hat{\mathcal{O}}_{V}+\hat{\mathcal{O}}_{\bar{V}} .
$$

The operator $\hat{\mathcal{O}}_{V}$ represents a part of the operator $\hat{\mathcal{O}}$ acting to the particle when it is in the spatial region $V$. The operator $\hat{\mathcal{O}}_{\bar{V}}$ represents the remaining part of the operator $\hat{\mathcal{O}}$. Any one-body operator which is local in space, $\hat{\mathcal{O}}^{(1)}=\sum_{i=1}^{N} \hat{o}^{(1)}\left(\mathbf{r}_{i}, \sigma_{i}\right)$, can be decomposed as

$$
\begin{aligned}
\hat{\mathcal{O}}^{(1)} & =\sum_{i=1}^{N}\left(\Theta_{V}\left(\mathbf{r}_{i}\right)+\Theta_{\bar{V}}\left(\mathbf{r}_{i}\right)\right) \hat{o}^{(1)}\left(\mathbf{r}_{i}, \sigma_{i}\right) \\
& =\hat{\mathcal{O}}_{V}^{(1)}+\hat{\mathcal{O}}_{\bar{V}}^{(1)},
\end{aligned}
$$

where $\sigma_{i}$ denotes the spin coordinate of a particle $i$. In the same way, a two-body operator, $\hat{\mathcal{O}}^{(2)}=$ $\sum_{i<j}^{N} \hat{o}^{(2)}\left(\mathbf{r}_{i}, \sigma_{i}, \mathbf{r}_{j}, \sigma_{j}\right)$, can be decomposed as

$$
\begin{aligned}
\hat{\mathcal{O}}^{(2)}=\sum_{i<j}^{N} & \left(\Theta_{V}\left(\mathbf{r}_{i}\right)+\Theta_{\bar{V}}\left(\mathbf{r}_{i}\right)\right)\left(\Theta_{V}\left(\mathbf{r}_{j}\right)+\Theta_{\bar{V}}\left(\mathbf{r}_{j}\right)\right) \\
& \times \hat{o}^{(2)}\left(\mathbf{r}_{i}, \sigma_{i}, \mathbf{r}_{j}, \sigma_{j}\right) \\
=\sum_{i<j}^{N}( & \Theta_{V}\left(\mathbf{r}_{i}\right) \Theta_{V}\left(\mathbf{r}_{j}\right)+\Theta_{\bar{V}}\left(\mathbf{r}_{i}\right) \Theta_{\bar{V}}\left(\mathbf{r}_{j}\right) \\
& \left.+\Theta_{V}\left(\mathbf{r}_{i}\right) \Theta_{\bar{V}}\left(\mathbf{r}_{j}\right)+\Theta_{\bar{V}}\left(\mathbf{r}_{i}\right) \Theta_{V}\left(\mathbf{r}_{j}\right)\right) \\
& \times \hat{o}^{(2)}\left(\mathbf{r}_{i}, \sigma_{i}, \mathbf{r}_{j}, \sigma_{j}\right) \\
= & \hat{\mathcal{O}}_{V}^{(2)}+\hat{\mathcal{O}}_{\bar{V}}^{(2)}+\hat{\mathcal{O}}_{V \bar{V}}^{(2)} .
\end{aligned}
$$

The first (second) term represents two-body interactions which act when both particles $i$ and $j$ are in the spatial region $V(\bar{V})$. The third term represents two-body interactions which act when a particle $i$ is in the spatial region $V$ and a particle $j$ is in the spatial region $\bar{V}$. For wave functions after collision in which two fragments are well separated, the third term can be ignored if the operator is short-range two-body interactions. When we calculate excitation energies of fragment nuclei, we ignore long-ranged Coulomb interactions acting protons belonging to different fragments.

The expectation value of the operator $\hat{\mathcal{O}}$ in the fragment which is composed of $n$ particles and locates in the spatial region $V$ is given by the expectation value of the operator $\hat{\mathcal{O}}_{V}$ in the wave function $\Psi_{n}$,

$$
\mathcal{O}_{n}^{V}=\frac{\left\langle\Psi_{n}\left|\hat{\mathcal{O}}_{V}\right| \Psi_{n}\right\rangle}{\left\langle\Psi_{n} \mid \Psi_{n}\right\rangle}
$$

The bracket $\left\langle\Psi_{n}\left|\hat{O}_{V}\right| \Psi_{n}\right\rangle$ is defined by

$$
\begin{aligned}
& \left\langle\Psi_{n}\left|\hat{\mathcal{O}}_{V}\right| \Psi_{n}\right\rangle \\
& \equiv \int d x_{1} \cdots \int d x_{N} \Psi_{n}^{*}\left(x_{1}, \cdots, x_{N}\right) \hat{\mathcal{O}}_{V} \Psi_{n}\left(x_{1}, \cdots, x_{N}\right),
\end{aligned}
$$


where the integral over $x$ includes an integration over space and a sum over spin states, $\int d x \equiv \sum_{\sigma} \int d \boldsymbol{r}$. Here and hereafter, we often use the bracket notation to simplify equations.

The expectation value of the operator $\hat{\mathcal{O}}_{V}$ without PNP is given by $\mathcal{O}_{V}=\left\langle\Psi\left|\hat{\mathcal{O}}_{V}\right| \Psi\right\rangle$. It is related to $\hat{\mathcal{O}}_{n}^{V}$ by

$$
\mathcal{O}_{V}=\sum_{n=0}^{N} P_{n} \mathcal{O}_{n}^{V}
$$

where $P_{n}$ is defined by $P_{n}=\left\langle\Psi_{n} \mid \Psi_{n}\right\rangle=\left\langle\Psi\left|\hat{P}_{n}\right| \Psi\right\rangle$. To derive Eq. (12), we used identities $\sum_{n=0}^{N} \hat{P}_{n}=1, \hat{P}_{n} \hat{P}_{n^{\prime}}=$ $\delta_{n n^{\prime}} \hat{P}_{n}$, and $\left[\hat{\mathcal{O}}_{V}, \hat{P}_{n}\right]=0$.

\section{B. Formulae for the Slater determinant}

We present formulae of expectation values which are useful for the TDHF wave function $\Psi$ given by a single Slater determinant composed of single-particle wave functions $\psi_{i}(x)$,

$$
\Psi\left(x_{1}, \cdots, x_{N}\right)=\frac{1}{\sqrt{N !}} \operatorname{det}\left\{\psi_{i}\left(x_{j}\right)\right\} .
$$

Using the PNP operator of Eq. (4), the probability $P_{n}$ can be calculated as [2, 5]

$$
\begin{aligned}
P_{n} & =\frac{1}{2 \pi} \int_{0}^{2 \pi} d \theta e^{\mathrm{i} n \theta}\left\langle\Psi\left|e^{-\mathrm{i} \hat{N}_{V} \theta}\right| \Psi\right\rangle \\
& =\frac{1}{2 \pi} \int_{0}^{2 \pi} d \theta e^{\mathrm{i} n \theta} \operatorname{det} \mathcal{B}(\theta) .
\end{aligned}
$$

$\mathcal{B}(\theta)$ denotes a $N$-dimensional matrix,

$$
(\mathcal{B}(\theta))_{i j}=\int d x \psi_{i}^{*}(x) \psi_{j}(x, \theta),
$$

where $\psi_{i}(x, \theta)$ is defined by

$$
\psi_{i}(x, \theta) \equiv\left(\Theta_{\bar{V}}(\mathbf{r})+e^{-\mathrm{i} \theta} \Theta_{V}(\mathbf{r})\right) \psi_{i}(x) .
$$

Using Eqs. (41) and (10), the expectation value $\mathcal{O}_{n}^{V}$ is expressed as

$$
\mathcal{O}_{n}^{V}=\frac{1}{2 \pi P_{n}} \int_{0}^{2 \pi} d \theta e^{\mathrm{i} n \theta}\left\langle\Psi\left|\hat{\mathcal{O}}_{V} e^{-\mathrm{i} \hat{N}_{V} \theta}\right| \Psi\right\rangle .
$$

In the case of one- and two-body operators, $\hat{\mathcal{O}}_{V}^{(1)}$ and $\hat{\mathcal{O}}_{V}^{(2)}$, in Eqs. (8) and (9), expectation values can be cal- culated by [15]

$$
\begin{aligned}
& \mathcal{O}_{n}^{V(1)}=\frac{1}{2 \pi P_{n}} \int_{0}^{2 \pi} d \theta e^{\mathrm{i} n \theta} \operatorname{det} \mathcal{B}(\theta) \\
& \times \sum_{i=1}^{N} \int_{V} d x \psi_{i}^{*}(x) \hat{o}^{(1)}(x) \tilde{\psi}_{i}(x, \theta), \\
& \mathcal{O}_{n}^{V(2)}=\frac{1}{2 \pi P_{n}} \int_{0}^{2 \pi} d \theta e^{\mathrm{i} n \theta} \operatorname{det} \mathcal{B}(\theta) \sum_{i<j}^{N} \\
& \times\left\{\int_{V} d x \int_{V} d x^{\prime} \psi_{i}^{*}(x) \psi_{j}^{*}\left(x^{\prime}\right) \hat{o}^{(2)}\left(x, x^{\prime}\right) \tilde{\psi}_{i}(x, \theta) \tilde{\psi}_{j}\left(x^{\prime}, \theta\right)\right. \\
& \left.-\int_{V} d x \int_{V} d x^{\prime} \psi_{i}^{*}(x) \psi_{j}^{*}\left(x^{\prime}\right) \hat{o}^{(2)}\left(x, x^{\prime}\right) \tilde{\psi}_{j}(x, \theta) \tilde{\psi}_{i}\left(x^{\prime}, \theta\right)\right\},
\end{aligned}
$$

where $\tilde{\psi}_{i}(x, \theta)$ is defined by

$$
\tilde{\psi}_{i}(x, \theta) \equiv \sum_{k=1}^{N} \psi_{k}(x, \theta)\left(\mathcal{B}^{-1}(\theta)\right)_{k i} .
$$

We note that $\left\{\tilde{\psi}_{i}\right\}$ are biorthonormal to $\left\{\psi_{i}\right\}$, i.e. $\int d x \psi_{i}^{*}(x) \tilde{\psi}_{j}(x, \theta)=\delta_{i j}$.

\section{Application to the TDHF wave function}

In actual TDHF calculations, the many-body wave function $\Psi$ is given by a product of two Slater determinants, $\Psi=\Psi_{\nu} \Psi_{\pi}$, where $\Psi_{\nu}$ is for neutrons and $\Psi_{\pi}$ is for protons. We present formulae of expectation values for this wave function. We denote the PNP operator for neutrons (protons) as $\hat{P}_{N}^{(n)}\left(\hat{P}_{Z}^{(p)}\right)$, where $N(Z)$ is the number of neutrons (protons) in the spatial region $V$. The probability that $N$ neutrons and $Z$ protons are in the spatial region $V$ is then given by a product of probabilities for neutrons and protons,

$$
\begin{aligned}
P_{N, Z} & =\left\langle\Psi\left|\hat{P}_{N}^{(n)} \hat{P}_{Z}^{(p)}\right| \Psi\right\rangle \\
& =\left\langle\Psi_{\nu}\left|\hat{P}_{N}^{(n)}\right| \Psi_{\nu}\right\rangle\left\langle\Psi_{\pi}\left|\hat{P}_{Z}^{(p)}\right| \Psi_{\pi}\right\rangle \\
& =P_{N}^{(n)} P_{Z}^{(p)} .
\end{aligned}
$$

We first consider expectation values for a one-body operator. We note that any one-body operator can be written as a sum of operators for neutrons and for protons, $\hat{\mathcal{O}}_{V}^{(1)}=\hat{\mathcal{O}}_{V}^{(1, n)}+\hat{\mathcal{O}}_{V}^{(1, p)}$. Thus the expectation value of the one-body operator $\hat{\mathcal{O}}_{V}^{(1)}$ is given by a sum of two terms. For the fragment nucleus specified by $N$ and $Z$, we have

$$
\begin{aligned}
\mathcal{O}_{N, Z}^{V(1)} & =\frac{\left\langle\Psi\left|\hat{\mathcal{O}}_{V}^{(1)} \hat{P}_{N}^{(n)} \hat{P}_{Z}^{(p)}\right| \Psi\right\rangle}{\left\langle\Psi\left|\hat{P}_{N}^{(n)} \hat{P}_{Z}^{(p)}\right| \Psi\right\rangle} \\
& =\frac{\left\langle\Psi_{\nu}\left|\hat{\mathcal{O}}_{V}^{(1, n)} \hat{P}_{N}^{(n)}\right| \Psi_{\nu}\right\rangle}{\left\langle\Psi_{\nu}\left|\hat{P}_{N}^{(n)}\right| \Psi_{\nu}\right\rangle}+\frac{\left\langle\Psi_{\pi}\left|\hat{\mathcal{O}}_{V}^{(1, p)} \hat{P}_{Z}^{(p)}\right| \Psi_{\pi}\right\rangle}{\left\langle\Psi_{\pi}\left|\hat{P}_{Z}^{(p)}\right| \Psi_{\pi}\right\rangle} \\
& =\mathcal{O}_{N}^{V(1, n)}+\mathcal{O}_{Z}^{V(1, p)}
\end{aligned}
$$


$\mathcal{O}_{n}^{V(1, q)}$ is defined by

$$
\mathcal{O}_{n}^{V(1, q)}=\frac{1}{2 \pi P_{n}^{(q)}} \int_{0}^{2 \pi} d \theta e^{\mathrm{i} n \theta}\left\langle\Psi_{q}\left|\hat{\mathcal{O}}_{V}^{(1, q)} e^{-\mathrm{i} \hat{N}_{V}^{(q)} \theta}\right| \Psi_{q}\right\rangle,
$$

where $\hat{N}_{V}^{(q)}$ denotes the particle-number operator for neutrons $(q=n)$ and for protons $(q=p)$ in the spatial region $V$. We will use these formulae, Eqs. (22) and (23), to calculate expectation values of the kinetic energy operator included in the Hamiltonian and of the angular momentum operator.

For a two-body operator, expectation values are not simply given by a sum of neutron and proton contributions, since two-body operators act between neutrons and protons. Therefore, we apply the PNP operators for both neutrons and protons simultaneously,

$$
\begin{array}{r}
\mathcal{O}_{N, Z}^{V(2)}=\frac{1}{(2 \pi)^{2} P_{N}^{(n)} P_{Z}^{(p)}} \int_{0}^{2 \pi} d \theta \int_{0}^{2 \pi} d \varphi e^{\mathrm{i}(N \theta+Z \varphi)} \\
\times\left\langle\Psi\left|\hat{\mathcal{O}}_{V}^{(2)} e^{-\mathrm{i}\left(\hat{N}_{V}^{(n)} \theta+\hat{N}_{V}^{(p)} \varphi\right)}\right| \Psi\right\rangle .
\end{array}
$$

We will use the above formula to evaluate excitation energy of nuclei produced through transfer processes.

To evaluate the excitation energy, we need to exclude the energy associated with the center-of-mass motion. For this purpose, we calculate the energy expectation value using Eqs. (21)-(24) in the coordinate system which moves with the fragment nucleus. In practice, we multiply all the single-particle wave functions by $e^{-\mathrm{i} \mathbf{K}_{\mu} \cdot \mathbf{r} / A_{\mu}}$, where $\mathbf{K}_{\mu}$ is given by $\mathbf{K}_{\mu}=M_{\mu} \dot{\mathbf{R}}_{\mu}\left(t_{f}\right) / \hbar$, with $M_{\mu}$, $A_{\mu}$, and $\dot{\mathbf{R}}_{\mu}\left(t_{f}\right)$ being the average mass, the average nucleon number, and the average velocity of the fragment $(\mu=$ PLF or TLF) in the spatial region $V$ at time $t_{f}$. We calculate the velocity of the fragment by $\dot{\mathbf{R}}_{\mu}\left(t_{f}\right) \equiv\left[\mathbf{R}_{\mu}\left(t_{f}+\Delta t\right)-\mathbf{R}_{\mu}\left(t_{f}-\Delta t\right)\right] /(2 \Delta t)$.

We denote the calculated energy expectation value in the fragment nucleus composed of $N$ neutrons and $Z$ protons as $\mathcal{E}_{N, Z}^{V}$. We separately achieve ground state calculations for the fragment nucleus composed of $N$ neutrons and $Z$ protons, which we denote as $E_{N, Z}^{\text {g.s. }}$. We evaluate an excitation energy of the fragment nucleus by

$$
E_{N, Z}^{* V}(E, b) \equiv \mathcal{E}_{N, Z}^{V}(E, b)-E_{N, Z}^{\text {g.s. }},
$$

where $E$ and $b$ denote the incident relative energy and the impact parameter, respectively.

In the ground state calculation, we employ a mass correction in the kinetic energy operator, $\frac{\hbar^{2}}{2 m} \rightarrow \frac{\hbar^{2}}{2 m}(1-$ $\left.\frac{1}{N+Z}\right)$, to take into account the center-of-mass correction. The same correction is applied in evaluating the expectation value of the kinetic energy operator using Eqs. (22) and (23), depending on numbers of neutrons and protons, $N$ and $Z$, in the fragment nucleus.

\section{AN ILLUSTRATIVE EXAMPLE: ${ }^{24} \mathrm{O}+{ }^{16} \mathrm{O}$ COLLISION}

To illustrate usefulness of the PNP method described in Sec. [II we analyze properties of fragment nuclei in ${ }^{24} \mathrm{O}+{ }^{16} \mathrm{O}$ collisions described by the TDHF theory. For ${ }^{24} \mathrm{O}$, pairing correlation may be important. In Ref. [16], the pairing interaction is reported to be negligible in the ground state, while finite contribution is reported in Ref. [17]. In this paper, we restrict ourselves to treatments ignoring pairing effects. We note that reactions including neutron-rich oxygen isotopes have been wellstudied in the TDHF theory as a typical reaction involving light unstable nuclei [18 21]. We will investigate expectation values of the angular momentum operator and average excitation energies.

We consider reactions in which two fragments are generated after collision. We call the ${ }^{24} \mathrm{O}$-like fragment nucleus as the PLF and the ${ }^{16} \mathrm{O}$-like fragment nucleus as the TLF. We describe the collision in the center-of-mass frame. We choose $x y$-plane as the reaction plane setting the incident direction parallel to the $x$ axis. The projectile, ${ }^{24} \mathrm{O}$, moves towards the negative- $x$ direction, while the target, ${ }^{16} \mathrm{O}$, moves towards the positive- $x$ direction. The impact parameter vector is set parallel to the positive- $y$ direction.

\section{A. Computational details}

We use our own computational code of TDHF calculation for nuclear collisions, as in Ref. [5]. Our code utilizes a three-dimensional uniform-grid representation for single-particle wave functions without any symmetry restrictions. The 11-point high-order finite difference formula is used for the spatial derivatives. For the time evolution, we use fourth order Taylor expansion method. The spatial grid points of $N_{x} \times N_{y} \times N_{z}=90 \times 80 \times 26$ are used with 0.8 -fm mesh spacing.

As an initial condition, two nuclei are placed at the distance of $32 \mathrm{fm}$ in the $x$ direction. The initial wave functions of projectile and target nuclei are prepared in a box with $N_{x} \times N_{y} \times N_{z}=40 \times 40 \times 26$ grid points. We calculate time evolution until a distance between the centers of the PLF and the TLF exceeds $32 \mathrm{fm}$. For the PNP analysis, integrals over $\theta$ are performed by employing the trapezoidal rule discretizing the interval $[0,2 \pi]$ into $M$ equal grids. We find that $M=30$ is sufficient for the ${ }^{24} \mathrm{O}+{ }^{16} \mathrm{O}$ system. All the results reported here are calculated using the Skyrme SLyIII.0.8 parameter set 22].

\section{B. Ground states}

We calculate ground states of ${ }^{16} \mathrm{O}$ and ${ }^{24} \mathrm{O}$ nuclei, which are both spherical in the self-consistent solutions. Figure 1 shows single-particle energies of neutrons (red solid lines) and protons (green dotted lines) in ${ }^{16} \mathrm{O}$ in 


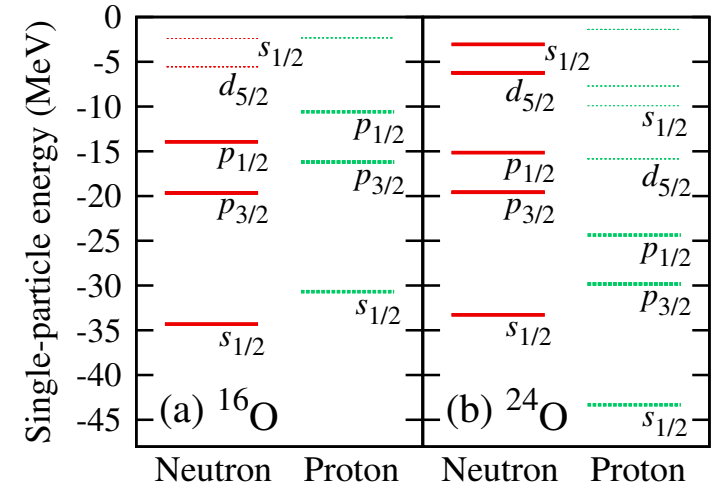

FIG. 1. (Color online) Single-particle energies of occupied orbitals for neutrons (thick red solid lines) and protons (thick green dotted lines) in ${ }^{16} \mathrm{O}$ and ${ }^{24} \mathrm{O}$ are shown in the panels (a) and (b), respectively. Single-particle energies of unoccupied orbitals are also shown by thin dotted lines.

panel (a) and in ${ }^{24} \mathrm{O}$ in panel (b). Occupied orbitals are shown by thick lines, while unoccupied orbitals are shown by thin lines. As recognized from the figure, there are neutron orbitals characterized by small binding energies in neutron-rich ${ }^{24} \mathrm{O}$ nucleus. All proton orbitals in ${ }^{24} \mathrm{O}$ are deeply bound.

\section{Reaction dynamics}

We first provide an overview of the reaction dynamics in ${ }^{24} \mathrm{O}+{ }^{16} \mathrm{O}$ collisions. In Fig. 2, the deflection angle $\Theta$ in the center-of-mass frame and the total kinetic energy loss (TKEL) are shown in the panels (a) and (b), respectively, as functions of the distance of closest approach, $d$. We evaluate $\Theta$ and TKEL from the momenta of two fragment nuclei and the Coulomb energy between them at the final stage of the TDHF calculation where two nuclei are well separated.

We employ the distance of closest approach $d$, instead of the impact parameter $b$. They are related by

$$
d=\frac{Z_{\mathrm{P}} Z_{\mathrm{T}} e^{2}}{2 E}+\sqrt{\left(\frac{Z_{\mathrm{P}} Z_{\mathrm{T}} e^{2}}{2 E}\right)^{2}+b^{2}}
$$

where $E$ denotes the incident relative energy. $Z_{\mathrm{P}}$ and $Z_{\mathrm{T}}$ denote the proton numbers of the projectile and the target, respectively. We consider it is useful to use $d$, because transfer reactions take place at around the distance of closest approach. For head-on collisions, calculated results are indicated by $b=0$ and are plotted against $d$ which is related to the incident relative energy $E$ by $d=Z_{\mathrm{P}} Z_{\mathrm{T}} e^{2} / E$.

We find the fusion reaction takes place at $d=9.4 \mathrm{fm}$ for head-on collision $(b=0)$ which corresponds to the incident energy of $E_{\text {lab }} \sim 24.5 \mathrm{MeV}$. For non-central collisions at incident energies of $E_{\text {lab }}=2,4$, and $8 \mathrm{MeV} /$ nucleon, the fusion reaction is found to take place at $d=8.7,8.3$, and $7.5 \mathrm{fm}$, respectively.

The deflection angle is positive for reactions at the incident energy of $2 \mathrm{MeV} /$ nucleon due to the Coulomb repul-

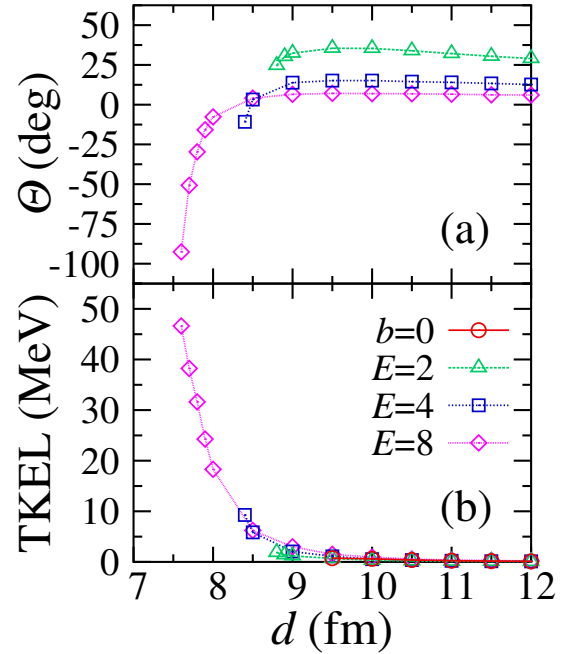

FIG. 2. (Color online) Deflection angle $\Theta$ in the center-ofmass frame (a) and total kinetic energy loss (b) are shown as functions of the distance of closest approach, $d=d(E, b)$.

sion, as seen in Fig. 2 (a). As the distance of closest approach decreases, the nuclear attractive interaction acts to decrease the deflection angle. It becomes negative for $d<8 \mathrm{fm}$ at the incident energy of $8 \mathrm{MeV} /$ nucleon. In the panel (b), we see an increase of the TKEL at the small- $d$ region where we observed negative deflection angles.

\section{Transfer probability}

In Fig 3, we show transfer probabilities calculated using Eq. (21). Red circles show probabilities for head-on collisions $(b=0)$ with several values of $d$. Green triangles, blue squares, and purple diamonds show probabilities as functions of $d$ for incident energies $E_{\text {lab }}=2,4$, and $8 \mathrm{MeV} /$ nucleon, respectively.

In the calculations, we adopted two choices for the spatial region $V$. For the probabilities observing a PLF, which are shown in the right panels of Fig. 3, we adopted a sphere with a radius of $16 \mathrm{fm}$ around the PLF for the spatial region $V$. For the probabilities observing a TLF shown in the left panels of Fig. 3, a sphere with a radius of $16 \mathrm{fm}$ around the TLF is used. We have confirmed that obtained results are almost independent of the chosen radius $R$ of the spatial region $V$, if $R$ is taken in the range of $15 \mathrm{fm}<R<20 \mathrm{fm}$. We will use this radius for evaluation of expectation values of angular momentum and excitation energies.

Figure 3 (a) and (b) show probabilities of one-proton transfer processes, while (c) and (d) show probabilities of two-proton transfer processes. We note that, from the above choices of $V$ for the PLF and the TLF, the probabilities of proton removal from ${ }^{16} \mathrm{O}((\mathrm{a})$ and (c)) should be coincide with the probabilities of proton addition to ${ }^{24} \mathrm{O}((\mathrm{b})$ and $(\mathrm{d})$ ), if the breakup processes can be neglected. As seen from the figure, (a) and (b) are very close to each other, indicating that the breakup processes 

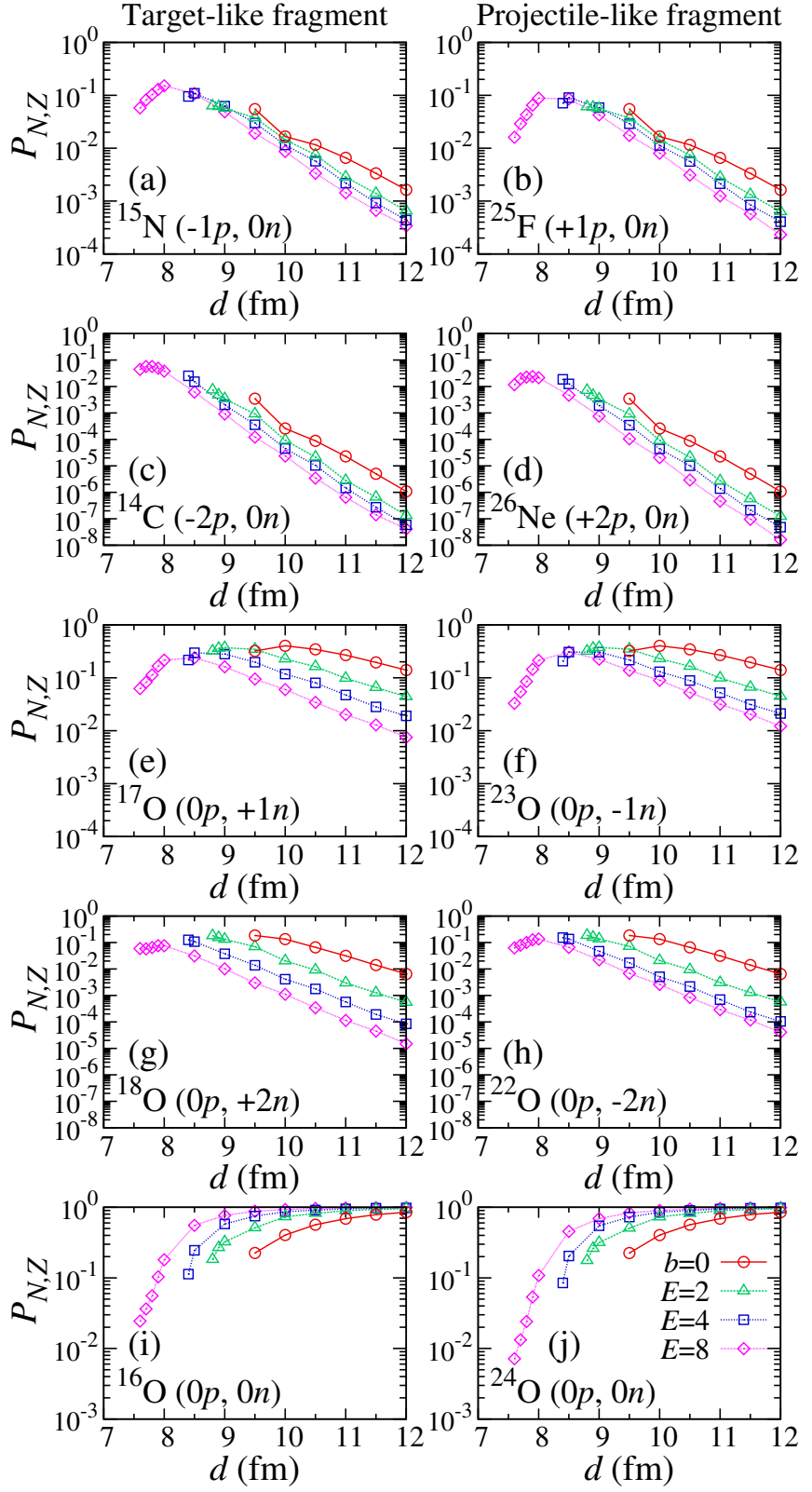

FIG. 3. (Color online) Transfer probabilities with respect to the TLF (left) and the PLF (right) are shown as functions of the distance of closest approach, $d=d(E, b)$.

are indeed negligible. We also find that (c) and (d) are close to each other. On the other hand, in the case of neutron transfer channels, one-neutron transfer in panels (e) and (f) and two-neutron transfer in panels (g) and (h), we find that the probability of neutron removal from ${ }^{24} \mathrm{O}$ is much larger than that of neutron addition to ${ }^{16} \mathrm{O}$, especially for reactions at $E_{\text {lab }}=8 \mathrm{MeV} /$ nucleon. This fact indicates that there are substantial probabilities of breakup processes for neutrons.

In Fig. 3. we find that transfer probabilities decrease as the incident energy increases. Comparing probabilities of neutron and proton transfer processes, neutron transfer probabilities are much larger than proton transfer probabilities at the same distance of closest approach and the same incident energy. We also find that the slope of probabilities for protons against the distance of closest approach is much steeper than that for neutrons. These features are consistent with orbital energies of the two colliding nuclei in their ground states which are shown in Fig. 1 Since there are neutrons bound weakly in ${ }^{24} \mathrm{O}$, transfer probabilities of neutrons are much larger than those of protons. Since these weekly bound neutrons are spatially extended in ${ }^{24} \mathrm{O}$, we find a long tail of neutron transfer probabilities.

At the highest incident energy of $E_{\text {lab }}=$ $8 \mathrm{MeV} /$ nucleon, the proton transfer probability is maximum around $d=8 \mathrm{fm}$. The probability decreases as the distance of closest approach decreases. The decrease at the small- $d$ region indicates the increase of probabilities for other channels with transfers of a larger number of protons.

\section{E. Angular momentum}

In this subsection, we investigate expectation values of the angular momentum operator in the fragment nuclei. We will use the same definition for $V$ as that in the previous subsection, spheres with a radius of $16 \mathrm{fm}$ around the center-of-mass of the PLF and the TLF. We consider the angular momentum operator in the spatial region $V, \hat{\mathbf{J}}_{V}=\hat{\mathbf{J}}_{V}^{(n)}+\hat{\mathbf{J}}_{V}^{(p)}$. The operator $\hat{\mathbf{J}}_{V}^{(q)}$ denotes the angular momentum operator for neutrons $(q=n)$ and for protons $(q=p)$ in the spatial region $V$, given by $\hat{\mathbf{J}}_{V}^{(q)}=\sum_{i \in q} \Theta_{V}\left(\mathbf{r}_{i}\right) \hat{\mathbf{j}}_{i}=\sum_{i \in q} \Theta_{V}\left(\mathbf{r}_{i}\right)\left[\left(\hat{\mathbf{r}}_{i}-\mathbf{R}_{\mu}\right) \times \hat{\mathbf{p}}_{i}+\hat{\mathbf{s}}_{i}\right]$. $\mathbf{R}_{\mu}$ is the center-of-mass coordinate of the fragment $(\mu=$ PLF or TLF).

Figure 4 shows expectation values of the angular momentum operators in the PLF and the TLF composed of specific numbers of neutrons and protons. A component perpendicular to the collision plane is shown. Left panels show expectation values in the TLF, while right panels show those in the PLF. For reactions at $E_{\text {lab }}=8 \mathrm{MeV} /$ nucleon, expectation values at the small- $d$ region, $d<8 \mathrm{fm}$, are always positive irrespective of the numbers of transferred nucleons. This fact supports a macroscopic picture of a friction converting the angular momentum from the nucleus-nucleus relative motion to the internal ones.

In the following, we discuss results at relatively large- $d$ region $(d>9 \mathrm{fm})$. In these reactions, the distance of closest approach is much larger than the sum of radii of two colliding nuclei, and transfer processes are considered to proceed as single-particle dynamics. TDHF calculations may describe either above-barrier transfer or quantum tunneling below the barrier. In nucleon removal channels ((a), (c), (f), and (h)), we find that the expectation values of the angular momentum operator are very small irrespective of either neutron(s) or proton(s) is(are) removed, either from ${ }^{16} \mathrm{O}$ or ${ }^{24} \mathrm{O}$. This fact may be understood from properties of orbitals. For ${ }^{16} \mathrm{O}$, orbitals of the smallest binding energy are $1 p_{1 / 2}$ for both neutrons 


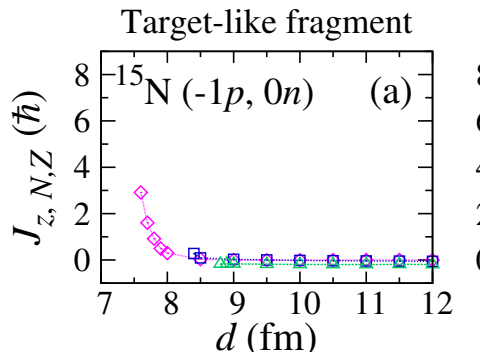

Projectile-like fragment
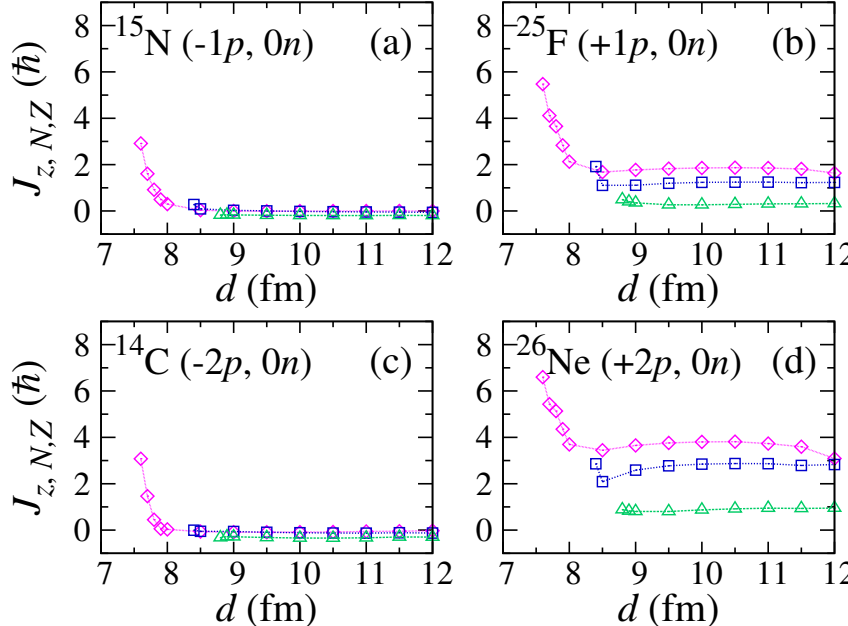

$d(\mathrm{fm})$
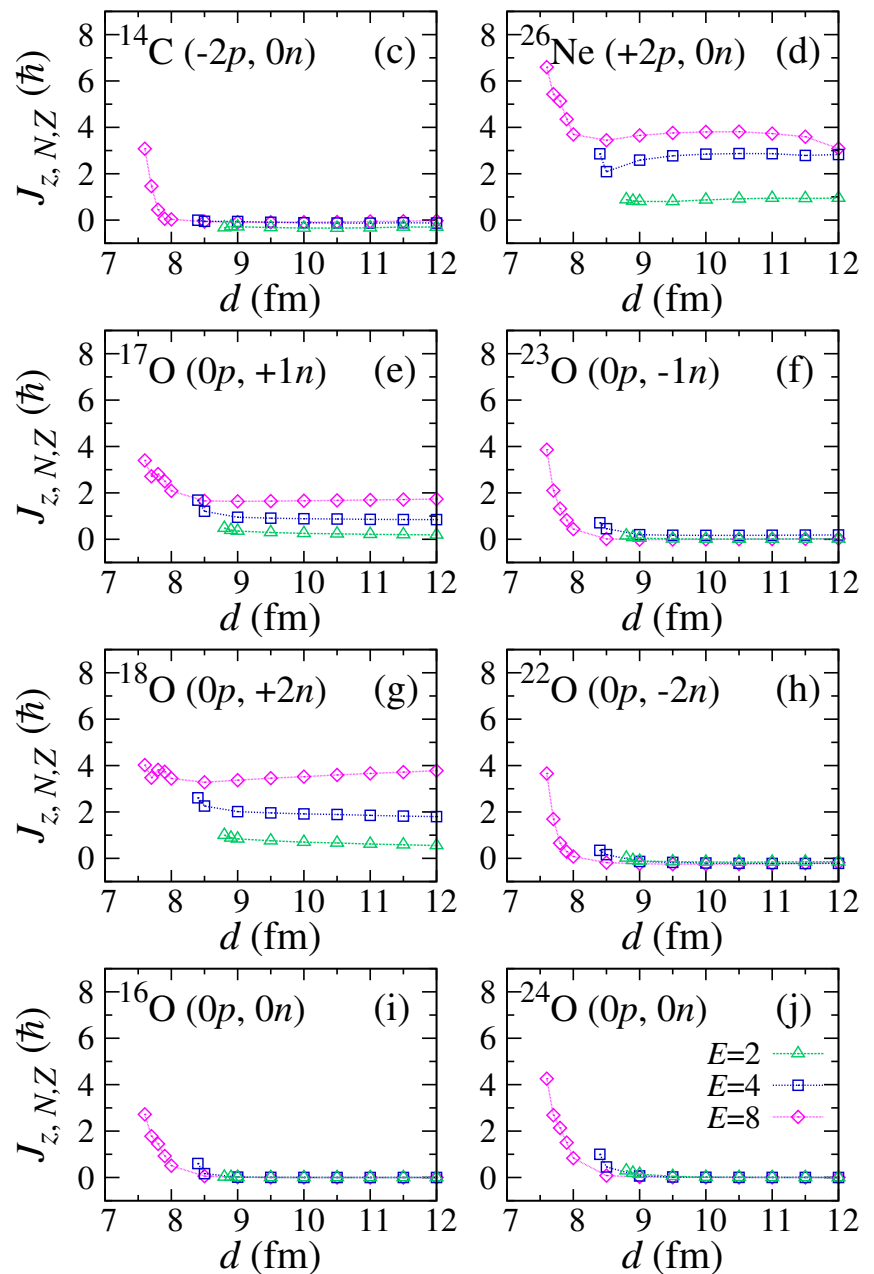

FIG. 4. (Color online) Expectation values of the angular momentum operator for fragment nuclei in each transfer channel are shown as functions of the distance of closest approach, $d=d(E, b)$.

and protons. For ${ }^{24} \mathrm{O}$, they are $2 s_{1 / 2}$ for neutrons and $1 p_{1 / 2}$ for protons. We thus find that the orbitals of the smallest binding energy are characterized by small angular momenta. Since nucleon removals from spatially extending single-particle orbitals are expected to take place for orbitals with the smallest binding energy, removal of nucleons from these orbitals may not leave large values of angular momentum in nucleon removed nuclei.

In nucleon addition channels ((b), (d), (e), and (g)), we find finite positive values of angular momentum in all channels. The expectation values increase as the incident energy increases. They do not depend much on the

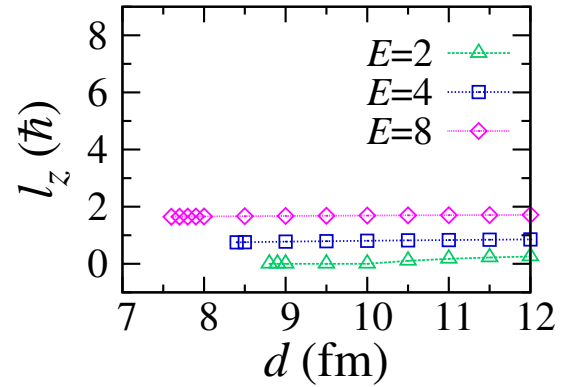

FIG. 5. (Color online) The angular momentum carried into ${ }^{16} \mathrm{O}$ by an added nucleon evaluated by Eqs. (27) and (28) is shown as a function of the distance of closest approach, $d=d(E, b)$.

distance of closest approach $d$. These features may be understood by the following intuitive considerations. Let us consider a transfer of one nucleon from ${ }^{24} \mathrm{O}$ to ${ }^{16} \mathrm{O}$. We assume that the nucleon transfer takes place when two nuclei are at the distance of closest approach. Ignoring the interaction potential by nuclear force, the relative velocity of two nuclei is approximately given by

$$
v_{\mathrm{rel}}=\sqrt{\frac{2}{\mu}\left(E-\frac{Z_{\mathrm{P}} Z_{\mathrm{T}} e^{2}}{d}\right)},
$$

where $E$ and $\mu$ denote the incident relative energy and the reduced mass, respectively. In the rest frame of ${ }^{16} \mathrm{O}$ nucleus, we assume that the transferred nucleon has the same velocity as the relative velocity $v_{\text {rel }}$, ignoring the internal motion in ${ }^{24} \mathrm{O}$. This may be reasonable, since we observed very small expectation values of the angular momentum in nucleon removed fragments, as seen in Fig. 4 (a), (c), (f), and (h). If the transferred nucleon stays at the surface of ${ }^{16} \mathrm{O}$, the transferred nucleon brings the angular momentum,

$$
l_{z}=R m v_{\mathrm{rel}}
$$

into ${ }^{16} \mathrm{O}$, where $m$ is the nucleon mass and $R$ is the radius of ${ }^{16} \mathrm{O}$ which we estimate by a simple formula, $R=r_{0} A^{1 / 3}$, with $r_{0}=1.2 \mathrm{fm}$ and $A=16$.

In Fig. [5. we show the angular momentum $l_{z}$ evaluated using Eqs. (27) and (28) as functions of the distance of closest approach $d$ for several energies. The estimated values of the angular momentum coincide quantitatively with the calculated results in channels of one-neutron addition to ${ }^{16} \mathrm{O}$, shown in Fig. 囵(e). The estimated angular momentum depends little on the distance of closest approach $d$, since the Coulomb potential in Eq. (27) gives only a minor effect except for a case of very low incident energy. The angular momentum is roughly proportional to the square root of the energy. In the case of twonucleon transfer, the angular momentum carried into ${ }^{16} \mathrm{O}$ is given by twice of $l_{z}$. This reasonably explains the observation in the panel $(\mathrm{g})$. 


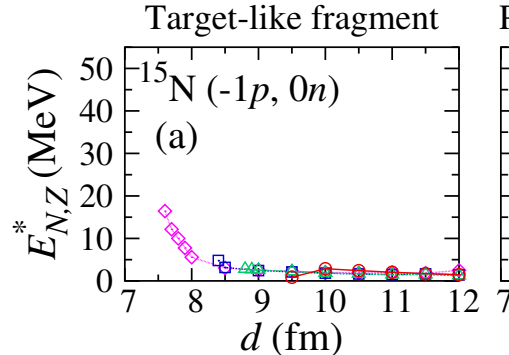

Projectile-like fragment
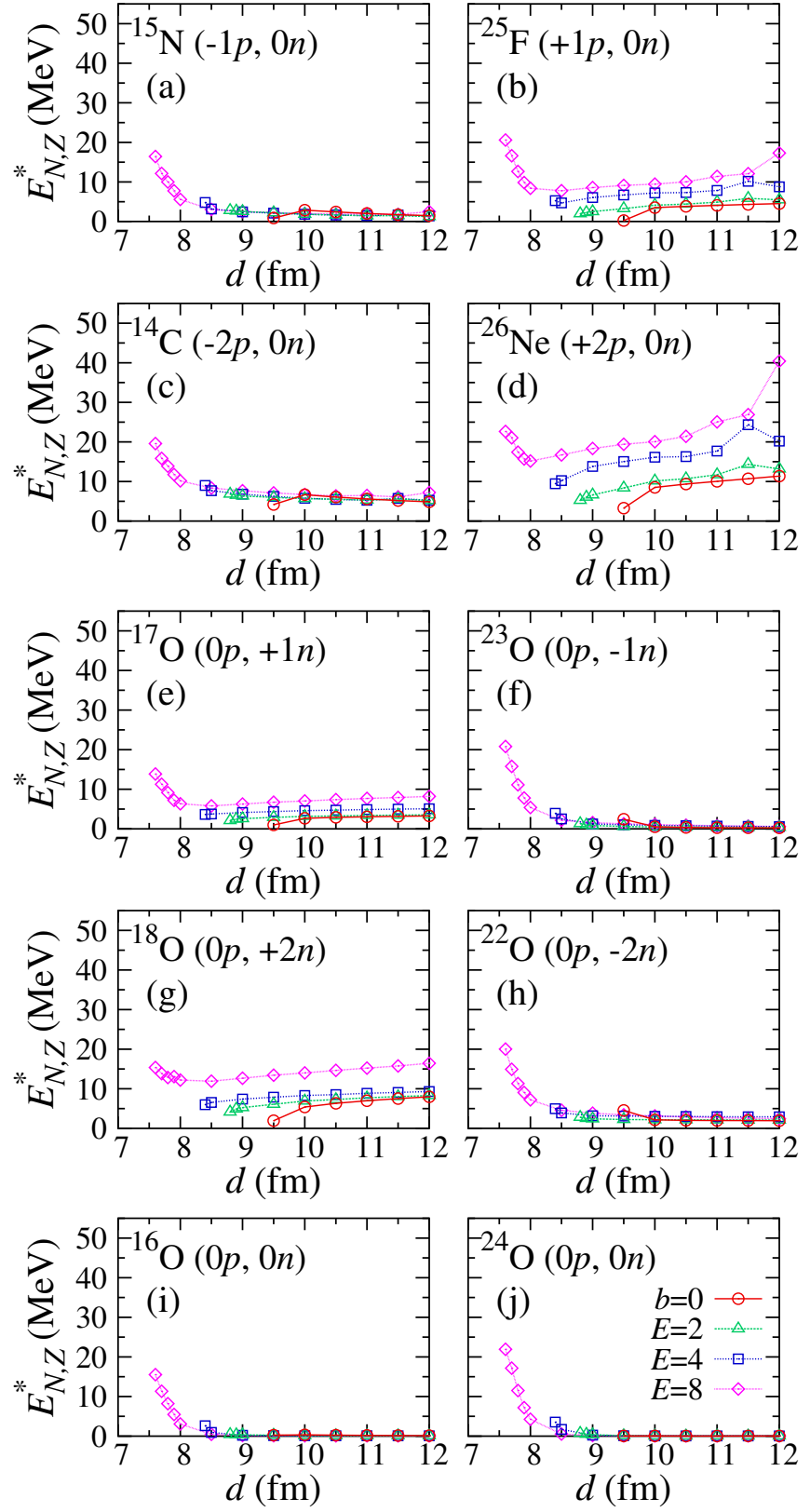

FIG. 6. (Color online) Average excitation energies of fragment nuclei in each transfer channel are shown as functions of the distance of closest approach, $d=d(E, b)$.

\section{F. Excitation energy}

In Fig. [6] we show excitation energies of fragment nuclei evaluated using Eq. (25) as functions of the distance of closest approach $d$. Left panels show the excitation energies of the TLF, while right panels show the excitation energies of the PLF. As in previous figures, there are two kinds of calculations: Red circles show results of head-on collisions $(b=0)$ varying the incident energy. Green triangles, blue squares, and purple diamonds show results for fixed incident energies, $E_{\text {lab }}=2,4$, and $8 \mathrm{MeV} /$ nucleon, respectively, changing the impact pa- rameter $b$.

As we mentioned below Eq. 25), we take into account the center-of-mass correction in calculating energies of fragment nuclei and reference energies of ground states in Eq. (25), while we ignore it in the time evolution calculations. For the quasi-elastic channels without nucleon transfer, we find very small average excitation energies at large- $d$ region, $d>9 \mathrm{fm}$, as shown in the panels (i) and $(\mathrm{j})$. This fact may indicate that the inconsistency between the treatments of the center-of-mass correction in evaluating excitation energies will not bring any serious problems.

In all channels, we find an increase of the excitation energy in a small- $d$ region, $d<8 \mathrm{fm}$, where we find an appreciable TKEL in Fig. 2 (b). At a large- $d$ region, $d>9 \mathrm{fm}$, we have found the small TKEL in Fig. 2(b). However, behavior of the excitation energy depends much on the transfer channels, as is evident from Fig. 6.

In nucleon removal channels ((a), (c), (f), and (h)), we find that excitation energies are rather small. In either one-neutron removal from ${ }^{24} \mathrm{O}$ in (f) or one-proton removal from ${ }^{16} \mathrm{O}$ in (a), the average excitation energy is less than $3 \mathrm{MeV}$. This indicates that the nucleon is removed dominantly from the highest occupied orbital. In two-nucleon removal channels ((c) and (h)), the excitation energy becomes somewhat large, about 5-10 MeV in two-proton removal from ${ }^{16} \mathrm{O}$ in (c). The excitation energies after nucleon removal are almost independent of the incident energy. This suggests that nucleons are removed gently even at higher incident energies.

Contrarily, in nucleon addition channels ((b), (d), (e), and $(\mathrm{g})$ ), we find that excitation energies depend much on the incident energy. A similar feature was also seen in the angular momentum shown in Fig. 4, where the added nucleon carries an angular momentum associated with the translational relative motion into the fragment. The expectation values of the angular momentum were also found to increase as the incident energy increases. This fact may be related to the increase of the excitation energies as the incident energy increases in nucleon addition channels: The transferred nucleons must stay at orbitals of higher angular momenta as the incident energy increases. The energies of orbitals with higher angular momenta are high.

For nucleon addition channels ((b), (d), (e), and (g)), we observe an increase of excitation energies as the distance of closest approach increases. One may consider that this fact contradicts to an intuitive picture that an excitation energy will be smaller as the distance of closest approach increases since two nuclei cannot collide violently. We examine this behavior for head-on collisions $(b=0)$. As shown by red circles in the panels $(\mathrm{b})$, (d), (e), and (g), the excitation energies are very small at $d=9.5 \mathrm{fm}$. This distance of closest approach corresponds to slightly outside the boundary of the fusion reaction. As the distance of closest approach increases (this corresponds to a decrease of the incident energy in the head-on collision), the excitation energies increase.

This puzzling behavior can be understood by the fol- 
lowing consideration. As we have shown in Fig. 1 the Fermi energies of neutrons and protons in ${ }^{24} \mathrm{O}$ and ${ }^{16} \mathrm{O}$ are rather different because of the excess neutrons in neutron-rich ${ }^{24} \mathrm{O}$. When a nucleon is transferred at a large distance of closest approach which is much larger than the sum of the radius of two colliding nuclei, the nucleon transfer is expected to take place between orbitals which are close in energy. The energy-conserving transfer processes must cause excitations of produced fragments if a neutron-rich nuclei is included in the collision.

Let us consider one-proton transfer from ${ }^{16} \mathrm{O}$ to ${ }^{24} \mathrm{O}$ in head-on collisions, which are shown by red circles in the panel (b). The transfer takes place dominantly for a proton in the highest occupied orbital of ${ }^{16} \mathrm{O}, 1 p_{1 / 2}$ at $-10.6 \mathrm{MeV}$ as shown in Fig. 1 (a). In Fig. 1 (b), we find proton orbitals at a similar orbital energy, $2 s_{1 / 2}$ at $-9.9 \mathrm{MeV}$. The proton highest occupied orbital of ${ }^{24} \mathrm{O}$ is $1 p_{1 / 2}$ at $-24.3 \mathrm{MeV}$ and there are $1 d_{5 / 2}$ unoccupied orbitals at $-15.8 \mathrm{MeV}$. Since one of the $1 d_{5 / 2}$ orbitals is occupied in the ground state of ${ }^{25} \mathrm{~F}$, we expect the excitation energy, $E^{*} \sim \varepsilon\left({ }^{24} \mathrm{O} ; \pi 2 s_{1 / 2}\right)-\varepsilon\left({ }^{24} \mathrm{O} ; \pi 1 d_{5 / 2}\right)=$ $5.9 \mathrm{MeV}$. This energy difference almost coincides with the average excitation energy of ${ }^{25} \mathrm{~F}$ shown in the panel (b) at the large- $d$ region.

We next consider one-neutron transfer from ${ }^{24} \mathrm{O}$ to ${ }^{16} \mathrm{O}$ in head-on collisions, which are shown by red circles in the panel (e). The highest occupied neutron orbital in ${ }^{24} \mathrm{O}$ is $2 s_{1 / 2}$ at $-3.1 \mathrm{MeV}$ as shown in Fig. 1 (b). In Fig. 1 (a), there are neutron unoccupied orbitals in ${ }^{16} \mathrm{O}$ at a similar energy, $2 s_{1 / 2}$ at $-2.4 \mathrm{MeV}$. Since the lowest neutron unoccupied orbital in ${ }^{16} \mathrm{O}$ is $1 d_{5 / 2}$ orbital at $-5.5 \mathrm{MeV}$ which is occupied in the ground state of ${ }^{17} \mathrm{O}$, we expect the excitation energy, $E^{*} \sim \varepsilon\left({ }^{16} \mathrm{O} ; \nu 2 s_{1 / 2}\right)-$ $\varepsilon\left({ }^{16} \mathrm{O} ; \nu 1 d_{5 / 2}\right)=3.1 \mathrm{MeV}$. This energy difference almost coincides with the average excitation energy of ${ }^{17} \mathrm{O}$ shown in the panel (e) at the large- $d$ region.

In the above considerations, we may understand the transfer mechanism in terms of orbital properties in the ground state: the highest occupied orbitals dominantly contribute to the transfer process. We note that, in Ref. 21], single-particle transfer dynamics in ${ }^{24} \mathrm{O}+{ }^{16} \mathrm{O}$ collision has been examined analyzing density contributions from individual orbitals. The result reported in Ref. 21] is consistent with the above conclusion.

We make a final comment on an abrupt increase of excitation energy seen at the largest $d$ value, $12 \mathrm{fm}$, and the highest incident energy, $8 \mathrm{MeV} /$ nucleon in panels (b) and (d). We consider that they are due to a numerical failure. We note that probabilities of these processes are very small, as confirmed in Fig. 3.

\section{SUMMARY}

In the time-dependent Hartree-Fock theory, low-energy heavy ion collisions are described by a time evolution of a single Slater-determinant wave function. At the final stage of calculation, the wave function may be regarded as a superposition of a number of channels with different particle numbers and quantum states. To obtain detailed information on reaction products, projection operator techniques will be useful. In this paper, we proposed a method to calculate expectation values of operators with the particle-number projection to investigate properties of projectile- and target-like fragments after collision.

To demonstrate usefulness of our method, we applied the method to one- and two-nucleon transfer processes in ${ }^{24} \mathrm{O}+{ }^{16} \mathrm{O}$ collisions. We analyzed expectation values of the angular momentum operator and average excitation energies of produced nuclei. For fragment nuclei after nucleon removal, we found small values of angular momentum and excitation energy, suggesting a gentle removal of nucleons. For fragment nuclei with added nucleons, we found substantial expectation values of angular momentum and average excitation energies. We have found that the expectation value of the angular momentum of produced nuclei is proportional to the relative velocity of the two colliding nuclei at the turning point. The excitation energy can be understood by a transfer of nucleons between approximately degenerate orbitals of projectile and target nuclei.

The above example clearly shows the usefulness of the present method for microscopic investigations of reaction mechanisms in heavy ion collisions. The formalism will also be useful to estimate effects of particle evaporation after multinucleon transfer processes, which are difficult to describe directly in the time-dependent Hartree-Fock calculation because of the very long timescale of the evaporation processes [23].

\section{ACKNOWLEDGMENTS}

K.S. would like to thank K. Washiyama and S.A. Sato for valuable comments and discussions. K.S. greatly appreciates the organization of the TALENT course \#6 "Theory for exploring nuclear reaction experiments" held at GANIL, Caen, France, in July 2013, which stimulates a part of analyses in the present work. This research used computational resources of the HPCI system provided by Information Initiative Center, Hokkaido University, through the HPCI System Research Project (Project ID: hp140010). This work was supported by the Japan Society of the Promotion of Science (JSPS) Grants-inAid for Scientific Research Grant Numbers 23340113 and 25104702 , and by the JSPS Grant-in-Aid for JSPS Fellows Grant Number 25-241. 
[1] C. Simenel, Eur. Phys. J. A 48, 152 (2012).

[2] C. Simenel, Phys. Rev. Lett. 105, 192701 (2010).

[3] M. Evers, M. Dasgupta, D.J. Hinde, D.H. Luong, R. Rafiei, R. du Rietz, and C. Simenel, Phys. Rev. C 84, 054614 (2011).

[4] G. Scamps and D. Lacroix, Phys. Rev. C 87, 014605 (2013).

[5] K. Sekizawa and K. Yabana, Phys. Rev. C 88, 014614 (2013).

[6] L. Corradi, J.H. He, D. Ackermann, A.M. Stefanini, A. Pisent, S. Beghini, G. Montagnoli, F. Scarlassara, G.F. Segato, G. Pollarolo, C.H. Dasso, and A. Winther, Phys. Rev. C 54, 201 (1996).

[7] L. Corradi, A.M. Stefanini, J.H. He, S. Beghini, G. Montagnoli, F. Scarlassara, G.F. Segato, G. Pollarolo, and C.H. Dasso, Phys. Rev. C 56, 938 (1997).

[8] L. Corradi, A.M. Vinodkumar, A.M. Stefanini, E. Fioretto, G. Prete, S. Beghini, G. Montagnoli, F. Scarlassara, G. Pollarolo, F. Cerutti, and A. Winther, Phys. Rev. C 66, 024606 (2002).

[9] S. Szilner, L. Corradi, G. Pollarolo, S. Beghini, B.R. Behera, E. Fioretto, A. Gadea, F. Haas, A. Latina, G. Montagnoli, F. Scarlassara, A.M. Stefanini, M. Trotta, A.M. Vinodkumar, and Y. Wu, Phys. Rev. C 71, 044610 (2005).

[10] A. Winther, Nucl. Phys. A572, 191 (1994); A594, 203
(1995).

[11] E. Vigezzi and A. Winther, Ann. Phys. (N.Y.) 192, 432 (1989).

[12] V. Zagrebaev and W. Greiner, J. Phys. G 31, 825 (2005).

[13] V. Zagrebaev and W. Greiner, J. Phys. G 34, 1 (2007).

[14] M. Bender, P.-H. Heenen, and P.-G. Reinhard, Rev. Mod. Phys. 75, 121 (2003).

[15] S. Shinohara, H. Ohta, T. Nakatsukasa, and K. Yabana, Phys. Rev. C 74, 054315 (2006).

[16] S. Hilaire and M. Girod, Eur. Phys. J. A 33, 237 (2007).; http://www-phynu.cea.fr/science_en_ligne/carte_ potentiels_microscopiques/carte_potentiel_nucleaire_eng.htm

[17] M. Tohyama and A.S. Umar, Phys. Lett. B 549, 72 (2002).

[18] K.-H. Kim, T. Otsuka, and M. Tohyama, Phys. Rev. C 50, R566 (1994).

[19] K.-H. Kim, T. Otsuka, and P. Bonche, J. Phys. G 23, 1267 (1997)

[20] A.S. Umar and V.E. Oberacker, Phys. Rev. C 73, 054607 (2006).

[21] A.S. Umar, V.E. Oberacker, and J.A. Maruhn, Eur. Phys. J. A 37, 245 (2008).

[22] K. Washiyama, K. Bennaceur, B. Avez, M. Bender, P.H. Heenen, and V. Hellemans, Phys. Rev. C 86, 054309 (2012).

[23] K. Sekizawa and K. Yabana, arXiv:1403.2862 [nucl-th]. 